\title{
Analysis on distribution features and drug resistance of clinically isolated Acinetobacter baumannii
}

\author{
GUANGMING REN, MIN ZHOU, NING DING，NING ZHOU and QINGLING LI \\ Department of Pneumology, The First People's Hospital of Xuzhou, Xuzhou, Jiangsu 221000, P.R. China
}

Received December 21, 2015; Accepted July 11, 2016

DOI: $10.3892 /$ etm.2016.3513

\begin{abstract}
The aim of the present study was to examine the clinical distribution and drug resistance of Acinetobacter baumannii infection, and provide evidence of clinical medication as well as the prophylax is for the treatment of drug resistance bacteria. In total, 306 Acinetobacter baumanniis selected from routine culture were collected between January 2012 and December 2013, to analyze the distributions among clinical specimens and wards and their drug resistance state. Of the 306 Acinetobacter baumanniis, the main distribution of specimens was sputum, accounting for $77.78 \%$. The distribution of administrative office was dominated by intensive care unit with a proportion of $40.0 \%$ in 2012 , which rapidly increased to $60.9 \%$ in 2013 , followed by neurosurgery, respiration medicine and orthopedics with proportions of 23,12 and $9.0 \%$ in 2012 and $9.71,8.74$ and $3.88 \%$ in 2013 , respectively. The Acinetobacter baumannii's drug resistance rate of Tazobactam and Piperacillin was increased from $68.0 \%$ in 2012 to $71.36 \%$ in 2013. At the same time, the drug resistance rate of imipenem was enhanced from $66.0 \%$ in 2012 to $72.81 \%$ in 2013. By 2013, the drug resistance rates of penbritin, ceftizoxime, cefotetan and macrodantin reached $\leq 100 \%$. In conclusion, Acinetobacter baumannii mainly causes respiratory tract infection with severe drug resistance. The drug resistance of Acinetobacter baumannii was mainly manifested as multidrug resistance or even pan-drug resistance with an obvious increasing trend of tolerance. Thus, it is necessary to prevent and treat nosocomial infection, to minimize usage of antibiotics and to standardize medical operating, to reduce the increase in persistence.
\end{abstract}

\section{Introduction}

Acinetobacter baumannii is a type of aerobic gram-negative bacilli and non-fermented saccharide (1). It is widely distributed in nature, hospital environments, skin surface of humans and

Correspondence to: Dr Min Zhou, Department of Pneumology, The First People's Hospital of Xuzhou, 19 Zhongshan North Road, Xuzhou, Jiangsu 221000, P.R. China

E-mail: moi159@163.com

Key words: Acinetobacter baumanii, clinical distribution, drug resistance organs such as respiratory, intestinal and urinary tract (2). It is also the main pathogenic bacteria of acquired infections in the hospital. In recent years, owing to the wide use of antibacterial drugs such as $\beta$-lactam antibiotics and cephalosporin antibiotics (3) and the popularization of invasive procedures, the multidrug-resistant Acinetobacter baumannii detection ratio has shown an increasing trend (4). Additionally, Acinetobacter baumannii has become the main pathogenic bacteria, causing fulminant epidemic infections in the hospital, creating difficulties in clinical treatment $(5,6)$.

Therefore, an analysis and comparison of the clinical distribution and drug resistance of Acinetobacter baumannii at The First People's Hospital of Xuzhou (Jiangsu, China) between 2012 and 2013 was conducted. The study aimed to provide guidance on rational drug use in clinic and identify methods that could be utilized in the prevention and treatment of drug-resistant strains.

\section{Materials and methods}

Source of bacterial strain. A total of 306 Acinetobacter baumanniis were isolated from sputum, blood and urine samples which were obtained from the patients from various clinical departments at The First People's Hospital of Xuzhou between January 2012 and December 2013. Repeated bacterial strains were obtained from the same site from the same patient were also screened out.

The present study was approved by the Ethics Committee of the First People's Hospital of Xuzhou. Written informed consent was obtained from all participants or their families prior to the study.

Identification of bacteria and drug sensitivity test. Fully-automatic microorganism identification and drug sensitivity analysis was conducted using VITEK 2 (bioMerieux, Marcy l'Étoile, France). The results of drug sensitivity were assessed according to the criteria of Clinical and Laboratory Standards Institute in 2010 (7).

Quality-control strains. Escherichia coli ATCC 25922 and Pseudomonas aeruginosa ATCC 27853 were used in the present study as a control.

Statistical analysis. WHONE 5.5 (WHO Collaborating Centre; http://www.whonet.org/) (8), provided by the drug-resistant 
Table I. Constituent ratio of the distribution of 306 Acinetobacter baumanii clinical specimens (\%).

\begin{tabular}{lrc}
\hline Specimens & Plants & Constituent ratio, \% \\
\hline Sputum & 238 & 77.78 \\
CSF & 23 & 7.52 \\
Wound secretion & 27 & 8.82 \\
Blood & 7 & 2.29 \\
Throat swab & 5 & 1.63 \\
BALF & 2 & 0.65 \\
Others & 4 & 1.31 \\
Total & 306 & 100.00 \\
\hline
\end{tabular}

CSF, cerebrospinal fluid; BALF, bronchoalveolar lavage fluid.

Table II. Constituent ratio of distribution of 100 Acinetobacter baumanii clinical department specimens in 2012.

\begin{tabular}{lrc}
\hline Wards & Plants & Constituent ratio, \% \\
\hline ICU & 40 & 40 \\
Brain surgery & 23 & 23 \\
Respiratory medicine & 12 & 12 \\
Orthopaedics & 9 & 9 \\
Brain medicine & 2 & 2 \\
Hematology & 3 & 3 \\
General surgery & 3 & 3 \\
Others & 8 & 8 \\
Total & 100 & 100 \\
\hline
\end{tabular}

ICU, intensive care unit.

monitor group of the World Health Organization, was used for statistical analysis.

\section{Results}

Distribution of specimens. Sputum had the highest detection ratio at $77.78 \%$, followed by wound secretion at $8.82 \%$, and cerebrospinal fluid at $7.52 \%$ (Table I).

Distribution of departments. The highest proportion of positive specimens was intensive care unit (ICU), accounting for $40.0 \%$ in 2012 and increased to $60.19 \%$ in 2013 , followed by neurosurgery with the proportion of $23 \%$ in 2012 and $8.74 \%$ in 2013. Respiration medicine followed these with a proportion of $12.0 \%$ in 2012 and $7.77 \%$ in 2013 (Tables II and III).

Drug sensitivity. The Acinetobacter baumannii drug resistance rate of Tazobactam and Piperacillin increased from $68.0 \%$ in 2012 to $71.36 \%$ in 2013 . At the same time, the drug resistance rate of imipenem was elevated from $66.0 \%$ in 2012 to $72.81 \%$ in 2013 . Additionally, by 2013 , the drug resistance
Table III. Constituent ratio of the distribution of 206 Acinetobacter baumanii clinical department specimens in 2013 (\%).

\begin{tabular}{lrc}
\hline Wards & Plants & Constituent ratio, \% \\
\hline ICU & 124 & 60.19 \\
Brain surgery & 18 & 8.74 \\
Respiratory medicine & 16 & 7.77 \\
Orthopaedics & 8 & 3.88 \\
Brain medicine & 5 & 2.43 \\
Hematology & 1 & 0.49 \\
General surgery & 6 & 2.91 \\
Others & 28 & 13.59 \\
Total & 206 & 100.00 \\
\hline
\end{tabular}

ICU, intensive care unit.

rates of penbritin, ceftizoxime, cefotetan and macrodantin reached $100 \%$ (Tables IV and V).

\section{Discussion}

Sputum specimens are the main source of Acinetobacter baumannii, with the highest rate of $77.78 \%$, indicating that Acinetobacter baumannii mainly results in respiratory tract infection, a finding that was consistent with earlier reports $(9,10)$. Other specimen sources included wound secretion, cerebrospinal fluid, blood and throat swab (1-3). Thus, Acinetobacter baumannii also leads to surgical site infection, secondary meningitis, bacteremia and systematic infections of other organs.

ICU had the highest detection ratio among all administrative offices for 2012 and 2013, with 40 and $60.19 \%$, respectively. The increase indicated that Acinetobacter baumannii was one of the main opportunistic pathogens of ICU infections. The majority of patients in ICU are in poor health owing to various diseases including cardiovascular disease and diabetes (11). They are required to remain in the ICU for a long period of time and are subject to hypoimmunity. They are also administered antibiotics with strong antimicrobial activity and wide antibacterial spectrum. Additionally, most patients with medical histories including being on a ventilator machine and undergoing tracheotomy, constitute risk factors of opportunistic pathogen infection $(11,12)$. The majority of patients recovering from brain surgery are required to remain in a reclining position and are weak in immunity. The Acinetobacter baumannii can result in secondary meningitis, and the positive specimens of cerebrospinal fluid from brain surgery were secondary to ICU. Most of the patients in the Department of Pneumology of The First People's Hospital of Xuzhou experience severe chronic respiratory disease. Such patients included transfer patients from rural environments who were prone to vartious basic diseases such as cardiovascular disease and diabetes and the medical history of using antibiotics informally over a long period of time (11). Some of these patients were hormone-dependent, malnourished and had a lack of immunity. Therefore, the Department of 
Table IV. A total of 100 Acinetobacter baumanii drug-sensitive rates (\%) in 17 types of antibacterial agents in 2012.

\begin{tabular}{|c|c|c|c|}
\hline & Sensitive & Intermediary & Drug resistant \\
\hline Antibacterial drugs & $\begin{array}{c}\text { Plants sensitive rate, } \\
\text { no. }(\%)\end{array}$ & $\begin{array}{c}\text { Plants intermediary rate, } \\
\text { no. }(\%)\end{array}$ & $\begin{array}{c}\text { Plants resistant rate, } \\
\text { no. }(\%)\end{array}$ \\
\hline Penbritin & (1) & (3) & (96) \\
\hline Ceftazidime & (16) & (3) & $(81)$ \\
\hline Gentamicin & (18) & (1) & (81) \\
\hline Macrodantin & $(0)$ & $(0)$ & $(100)$ \\
\hline Amikacin & (74) & (3) & (23) \\
\hline Penbritin-S & $(18)$ & (2) & $(80)$ \\
\hline Levofloxacin & (18) & (8) & (74) \\
\hline Cefepime & (17) & (5) & (78) \\
\hline Tobramycin & (20) & $(0)$ & $(80)$ \\
\hline Ceftizoxime & $(0)$ & (1) & (99) \\
\hline CPFX & $(17)$ & (1) & $(82)$ \\
\hline Ceftriaxone & (2) & (3) & $(95)$ \\
\hline Cefotetan & $(0)$ & $(0)$ & $(100)$ \\
\hline PIPC+TAZ & (22) & (10) & $(68)$ \\
\hline Selectrin & (13) & $(0)$ & (87) \\
\hline Imipenem & (31) & (3) & (66) \\
\hline Aztreonam & (22) & (13) & $(85)$ \\
\hline
\end{tabular}

CPFX, ciprofloxacin; PIPC, piperacillin; TAZ, tazobactam.

Table V. A total of 206 Acinetobacter baumanii drug-sensitive rates (\%) in 17 types of antibacterial agents in 2013.

\begin{tabular}{|c|c|c|c|}
\hline & Sensitive & Intermediary & Drug resistant \\
\hline Antibacterial drugs & $\begin{array}{c}\text { Plants sensitive rate, } \\
\text { no. }(\%)\end{array}$ & $\begin{array}{c}\text { Plants intermediary rate, } \\
\text { no. }(\%)\end{array}$ & $\begin{array}{c}\text { Plants resistant rate, } \\
\text { no. }(\%)\end{array}$ \\
\hline Penbritin & $0(0)$ & $0(0)$ & $206(100)$ \\
\hline Ceftazidime & $24(11.65)$ & $9(4.37)$ & $173(83.98)$ \\
\hline Gentamicin & $32(15.53)$ & $1(0.49)$ & $173(83.98)$ \\
\hline Macrodantin & $0(0)$ & $0(0)$ & $206(100)$ \\
\hline Amikacin & $122(59.22)$ & $32(15.53)$ & $52(25.24)$ \\
\hline Penbritin-S & $32(15.53)$ & $2(0.97)$ & $172(83.50)$ \\
\hline Levofloxacin & $28(13.59)$ & $8(3.88)$ & $170(82.53)$ \\
\hline Cefepime & $27(13.11)$ & $5(2.43)$ & $174(84.46)$ \\
\hline Tobramycin & $37(17.96)$ & $0(0)$ & $169(82.04)$ \\
\hline Ceftizoxime & $0(0)$ & $0(0)$ & $100(100)$ \\
\hline CPFX & $23(11.16)$ & $1(0.49)$ & $182(88.35)$ \\
\hline Ceftriaxone & $2(0.97)$ & $19(9.22)$ & $185(89.81)$ \\
\hline Cefotetan & $0(0)$ & $0(0)$ & $206(100)$ \\
\hline PIPC+TAZ & $38(18.45)$ & $21(10.19)$ & $147(71.36)$ \\
\hline Selectrin & $35(16.99)$ & $0(0)$ & $171(83.01)$ \\
\hline Imipenem & $51(24.76)$ & $5(2.43)$ & $150(72.81)$ \\
\hline Aztreonam & $0(0)$ & $15(7.28)$ & $191(92.72)$ \\
\hline
\end{tabular}

CPFX, ciprofloxacin; PIPC, piperacillin; TAZ, tazobactam.

Pneumology is also one of the main departments that produce Acinetobacter baumannii.
Acinetobacter baumannii is a common opportunistic pathogen present in the hospital. It has a strong adaptive 
capacity to the environment and acquisition of exogenous drug resistance genes. Acinetobacter baumannii readily forms multidrug-resistant strains and spreads rapidly. Consequently, it has received attention worldwide. According to investigations conducted in 2012, the Acinetobacter baumannii drug-resistant rates of 16 types of antibiotics such as imipenem, piperacillin, tazobactam, cefepime, ciprofloxacin and ceftriaxone exceeded $60 \%$, with the exception of the drug-resistant rate of amikacin (23\%). Compared with 2012, besides the slight decrease of the drug-resistant rates of ceftriaxone and selectrin, the remaining 15 types of antibiotics all increased to some extent by 2013. Furthermore, the drug resistance rates of penbitrin, ceftizoxime, cefotetan and macrodantin all reached $\leq 100 \%$ by 2013 . In recent years, multidrug- and pan-drug resistant Acinetobacter baumannii have gradually become global clinical problems $(13,14)$. Most Acinetobacter baumannii under examination between 2012 and 2013 were multiple resistant bacteria, even pan-resistant bacteria, which resulted in serious problems in clinical treatment. According to relevant reports (15), the resistance mechanism of Acinetobacter baumanniito medicine is complicated. In general, it can generate $\beta$-lactamase to damage the structure of $\beta$-lactams, and change the quantities and structure of its own membrane protein to alter permeability. The variation of drug target penicillin-binding proteins cause the decrease of affinity to medicine. Additionally, the reduction of membrane protein permeability outside bacteria and the hyperfunction of the efflux pump system may reduce the effective drug level inside bacteria. Due to the wide use of broad-spectrum antibiotics, multidrug-resistant Acinetobacter baumannii increases constantly. Therefore, using antibiotics more canonically and reasonably is extremely important in controlling the development of bacterial drug resistance and protecting the effective antibacterial function of antibiotics.

In recent years, the drug-resistant ability of Acinetobacter baumannii to carbapenems has led to numerous infections in the hospital and poor prognosis. Thus, consecutive monitoring of Acinetobacter baumannii's drug resistance and drug sensitivity in the hospital should be investigated, the use of clinical antibiotics minimize, and close cooperation with infected patients should occur, together with enhancing the sanitary control of medical workers, disinfecting various medical equipment after use, and regularizing the diagnosis and treatment for reducing the infection of Acinetobacter baumannii $(16,17)$. Strict measures for controlling the increase of drug-resistant strains should be developed to reduce the prevalence and case fatality rates of infection in the hospital. Thus, understanding the drug resistance of Acinetobacter baumannii at various time points is significant in the application of clinical antibiotics to promote the result of clinical anti-infection treatment effectively.

\section{References}

1. Giamarellou H, Antoniadou A and Kanellakopoulou K: Acinetobacter baumannii: A universal threat to public health? Int J Antimicrob Agents 32: 106-119, 2008.

2. Mishra SK, Rijal BP and Pokhrel BM: Emerging threat of multidrug resistant bugs-Acinetobacter calcoaceticus baumannii complex and methicillin resistant Staphylococcus aureus. BMC Res Notes 6: 98, 2013.

3. Krcmery V and Kalavsky E: Multidrug-resistant Acinetobacter baumannii. Emerg Infect Dis 13: 943-944, 2007.

4. Zhu X, Zhang M, Wang S, et al: The clinical distribution and drug-resistant analysis of baumanii's infection in the hospital. Lab Med 27: 788-790, 2012 (In Chinese).

5. Gootz TD and Marra A: Acinetobacter baumannii: An emerging multidrug-resistant threat. Expert Rev Anti Infect Ther 6: 309-325, 2008.

6. Du J and Li W: Distribution and resistance analysis of 148 Acinetobacter baumannii clinical isolates. Lab Med Clinic 10: 155-156, 2013.

7. Hsueh PR, Ko WC, Wu JJ, Lu JJ, Wang FD, Wu HY, Wu TL and Teng LJ: Consensus statement on the adherence to Clinical and Laboratory Standards Institute (CLSI) Antimicrobial Susceptibility Testing Guidelines (CLSI-2010 and CLSI-2010update) for Enterobacteriaceae in clinical microbiology laboratories in Taiwan. J Microbiol Immunol Infect 43: 452-455, 2010.

8. Mochizuki T, Okamoto N, Yagishita T, Takuhiro K, Mashiko K, Ogawa F, Tosaka N, Kurokawa A and Yamamoto Y: Analysis of antimicrobial drug resistance of Staphylococcus aureus strains by WHONET 5: Microbiology laboratory database software. J Nippon Med Sch 71: 345-351, 2004.

9. Luo P, Dai W and Zhang L: Clinical distribution and drug resistance analysis of 1582 Acinetobacter baumannii. Chongqing Med 40: 224-228, 2011 (In Chinese).

10. Zhang Y, Jia W and Zhao Z: The clinical distribution and drug-resistant analysis of baumaniis. Chin J Nosocomiol 21: 364-366, 2011.

11. Feng D, Dong $\mathrm{F}$ and $\mathrm{Xu} \mathrm{X}$ : The clinical distribution and drug-resistant analysis of the baumanii in PICU. J Xinxiang Med Coll 25: 591-593, 2008 (In Chinese).

12. Xu G and Long X: The diagnoses of preventive antibacterials among 570 perioperative patients. J Pract Clin Med 12: 83-84, 2008.

13. Peleg AY, Seifert H and Paterson DL: Acinetobacter baumannii: Emergence of a successful pathogen. Clin Microbiol Rev 21: 538-582, 2008.

14. Gould IM: The epidemidogy of antibiotic resistance. Int $\mathrm{J}$ Antimicrob Agents (Suppl 1) 32: S2-S9, 2008.

15. Zhu M, Sun X and Jiang R: The research of multi-drug resistant baumanii's resistance mechanism to 7 kinds of antibacterial agents. Chin J Nosocomiology 20: 2184-2188, 2010.

16. Mendes RE, Bell JM, Turnidge JD, Castanheira $M$ and Jones RN: Emergence and widespread dissemination of OXA-23, $-24 / 40$ and -58 carbapenemases among Acinetobacter spp. in Asia-Pacific nations: Report from the SENTRY Surveillance Program. J Antimicrob Chemother 63: 55-59, 2009.

17. Lai Y, Wu J and Yang J: The monitor of clinical distribution and drug resistantances of baumanii. China Trop Med 12: 342-344, 2013 (In Chinese). 